\title{
An experimental study to investigate hydraulic fracture reorientation with respect to the principal stresses
}

\author{
A. Gotti, S. H. Fallahzadeh \& V. Rasouli \\ Curtin University, Western Australia
}

\begin{abstract}
This study investigated the propagation of an induced hydraulic fracture experimentally. A true triaxial stress cell (TTSC) was used to apply three independent stresses of vertical, maximum horizontal and minimum horizontal to a cube of tight sandstone $100 \mathrm{~mm}$ in size. A large stress anisotropy was applied in order to help the fracture to initiate more easily. Injection of the fracturing fluid was possible through a hole drilled at the centre of the sample which was plugged in top and bottom sections to give an openhole length of approximately $40 \mathrm{~mm}$ in the sample centre. A pronounced notch was created in the direction of minimum horizontal stress, i.e. different than the direction at which the fracture intends to open naturally. The hydraulic fracturing tests were conducted on four different samples with different locations for the notch to investigate how the fracture would propagate from different starting angles.

The results indicated that while the fracture initiates along the direction of the notch, it tends to rotate towards the maximum stress direction as it moves away from the wellbore wall. As a fracture is propagating through rock, it passes through inhomogeneities which cause a deviation in the propagation direction. The results showed that a fracture will still reorient to the direction of maximum stress after passing inhomogeneities not just at the wellbore wall, but throughout the rock. The complexity of hydraulic fracturing became apparent in this experiment as even in a highly controlled laboratory environment, some fractures propagated in directions that were unexpected. The presented results demonstrate the need for having a good understanding about the state of stresses when designing a hydraulic fracturing job for the field.

Keywords: true triaxial stress cell, hydraulic fracturing, fracture propagation direction, in-situ stresses, fracture reorientation.
\end{abstract}




\section{Introduction}

Hydraulic fracturing is a stimulation technique which is widely used to enhance production, in particular from unconventional reservoirs such as shale gas, tight formations and coalbed methane. As explained by Valko and Economides [1], the technique of hydraulic fracturing has allowed for low permeability reservoirs which would otherwise not be viable, to become economically producible. In a hydraulic fracturing design several parameters are involved; such as formation mechanical properties, e.g. elastic and strength properties, injecting fluid rheology and the state of principal stresses in the field. Initiation pressure required to open the formation in the vicinity of the wellbore wall is a function of stress anisotropy. Once the fracture initiated its propagation pressure is not only a function of in-situ stresses but also is influenced by the friction along the drill pipes, perforation tunnels and the pressure to keep the fracture open. The propagation direction of the induced fracture is dominantly along the direction of maximum stress, i.e. perpendicular to the least resistance force as shown by Hossain et al. [2].

The results of past studies have indicated that the propagation of a fracture plane may deviate from the maximum stress direction when the ratio of stress anisotropy is low [3]. Theoretically, there is no preferred fracture direction when the induced stresses around the wellbore are isotropic [4]. In low stress anisotropy, the propagation direction of a fracture is more influenced by the rock inhomogeneity as shown by Rasouli et al. [5]. A fracture around a wellbore tends to initiate from any weak point within the formation at the wellbore wall but will deviate towards the maximum stress direction as it becomes farther away from the wellbore and senses the in-situ stresses. This is the reason for having deviated fracture geometries at field scales when the fracture plane propagates tens of meters long.

In order for a fracture to open unnaturally and in the direction of minimum stress, a notch or inhomogeneity must be in place to assist the opening of the fracture. If no notch or inhomogeneity exists, the fracture will always open in a direction perpendicular to the minimum stress. Commonly used equations are used to theoretically calculate the fluid pressure that is required to fracture a sample at different angles around the wellbore that is under stress shown by Hudson and Harrison [6]. To initiate the fracture at the wellbore wall, the pressure required must be high enough to overcome the tensile strength of the rock plus the minimum effective stress.

Using the identical values for the tensile strength of the rock (TS), the pore pressure $\left(\mathrm{P}_{\mathrm{p}}\right)$ and the maximum and minimum stresses $\left(\sigma_{\mathrm{H}}\right.$ and $\left.\sigma_{\mathrm{h}}\right)$, it can be shown that the solved value of $\sigma_{\theta}$ will always be lower in the direction of maximum stress. This is why a fracture will always naturally open in the maximum stress direction since a lower initiation pressure is required.

Evans and Rasouli [7] have shown the applications of the true triaxial stress cell to allow for laboratory simulations of hydraulic fractures and for monitoring propagation. This study explores the use of the true triaxial stress cell to investigate how a hydraulic fracture will reorient from its initiation direction 
with respect to the principal stresses. The following sections will explain the process of sample preparation, set up of the sample into the true triaxial stress cell (TTSC) which was used for the experiments and the results of the experiments.

\section{Sample preparation}

Four samples were tested in this study. Sample one was prepared by firstly cutting a section of sandstone to a cube $100 \mathrm{~mm}$ in size. The sandstone used was well consolidated and had a very low permeability of approximately $3 \mathrm{mD}$. A low permeability rock sample is critical for the experiment to ensure that there is minimal fracture fluid loss into the pores of the rock (i.e. leak-off) during fracturing. A $6 \mathrm{~mm}$ drill bit was then used to drill a hole through the centre of the sample. Once drilled, two notches were created on opposite sides in the drilled hole along the planned direction of maximum stress. Notches were created to assist the fracture to initiate around the borehole.

Sample one was created as a base case to ensure the fracture would open in the direction of the notches. Once the notches were created, a $30 \mathrm{~mm}$ long solid metal rod of $5 \mathrm{~mm}$ in diameter was cut and used as a plug for the bottom of the sample. High strength adhesive glue was used to coat the metal rod and seal it at the bottom of the drilled hole in the sample. This glued rod acts as a packer to ensure injected fluid does not escape through the sample. Once in place, a metal pipe $5 \mathrm{~mm}$ in diameter and $300 \mathrm{~mm}$ long was glued $30 \mathrm{~mm}$ in from the top of the sample. This metal rod provided a connection for the injection fluid to flow through to the $40 \mathrm{~mm}$ of remaining open hole in the sample.

A small open hole section is left in the centre of the sample as having a small open hole section allows for a larger distance before the nearest boundary is met, at which point the fracture will stop propagating. The sample was then left to rest for 2 days to ensure the glue had set completely before being loaded into the TTSC. Figure 1 shows the $100 \mathrm{~mm}$ cube sample (left) and the drilled hole at its centre (middle) and the connection pipe in place (right).

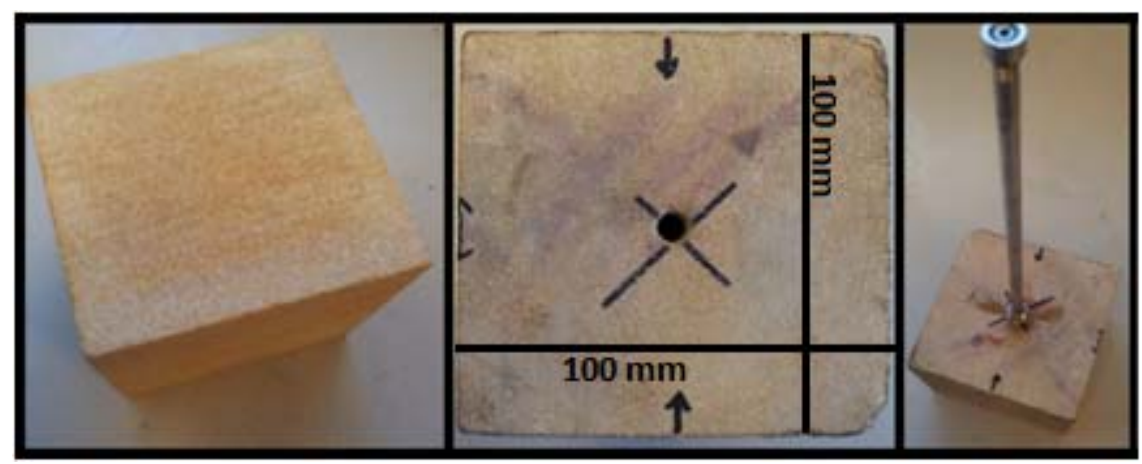

Figure 1: A 100mm sample (left) with a drilled hole at its centre (middle) and the connection pipe for fluid injection (right) was used for hydraulic fracturing experiment. 
Sample two was prepared in a similar way to sample one but the minimum stress in the TTSC, as will be explained in the next section was applied along the direction of the notches: this was to observe the tendency of the fracture to reorient to the maximum stress direction after propagating some distance apart from the wellbore wall. The largest deviation from the direction of maximum stress was chosen to provide results of a case where it would be most difficult for the fracture to curve back to the direction of maximum stress. This sample was completed in the same way as sample one, with a $40 \mathrm{~mm}$ open hole section left in the centre of the sample between the two metal rods which seal the open hole section.

The third sample used for the experiment in this study was the already fractured sample two. The idea was to apply a large maximum stress perpendicular to the opened fracture plane and repeat the hydraulic fracturing test. High strength adhesive and impermeable glue was used to re-seal the opened fracture, but leaving a $10 \mathrm{~mm}$ unglued section on both fracture wings around the wellbore open hole section. The result was two fracture wings $10 \mathrm{~mm}$ $\mathrm{x} 40 \mathrm{~mm}$ in area on each side of the wellbore, with the rest of the fracture being completely sealed by the glue. This in turn created a large notch or crevice to assist the fracture to open in the direction of minimum stress with the idea of observing any reorientation for the fracture once initiated around the borehole. The sample was put under a small amount of stress for three hours after the adhesive was applied to ensure the fracture was forced closed and so the glue would seal it consistently. Once the adhesive had set, a quick injection test was done to ensure that there were no leaks and so the sample would be ready for another fracture test.

Sample four was prepared differently after learning from the results of the first three samples. A different material was also chosen for the fourth test to compare results with how the fracture propagated though the sandstone. This sample was prepared in to a $100 \mathrm{~mm}$ cube to keep constant with the other samples. Rather than drill through the entire sample and then require a plug at the bottom, this sample was only drilled $70 \mathrm{~mm}$ down, leaving $30 \mathrm{~mm}$ of the sample undrilled. It was also learned from previous tests that the fracture appears to reach the top of the sample before the bottom of the sample so the open hole area was adjusted in this sample to be slightly closer to the bottom of the sample to allow more time for a fracture to propagate before reaching the first boundary. A greater focus was placed on the creation of the notches with this sample as it was identified from previous tests that the notch size needed to be increased. To create the notch to be deeper, a much smaller drill bit was used to shave the sides of the wellbore from inside the hole which was more effective than using metal rods and other tools to manually scratch the wellbore wall to create a notch or crevice. Once the notches had been made at an angle of 45 degrees to the direction of maximum stress, the rod that then connects to the hydraulic fracturing fluid chamber was inserted and glued. 


\section{Sample set up in TTSC}

There are two main components to the TTSC for hydraulic fracturing tests which include the cell itself and the hydraulic fracturing fluid chamber which is a separate piece of equipment that is not directly connected to the TTSC. The TTSC is capable of supporting cube samples of up to $300 \mathrm{~mm}$, by using different sized surrounding aluminium blocks to bridge the gap depending on the sample size. The stress through each of the rams is powered by a piston which compresses the rams against the blocks surrounding the sample as oil is injected until the desired pressure has been reached. A very thin layer of lubricant was used between the ram and the blocks to ensure that the only stresses being applied were the principal stresses and that the blocks were not applying any shear stress to the sample which would negatively impact the results.

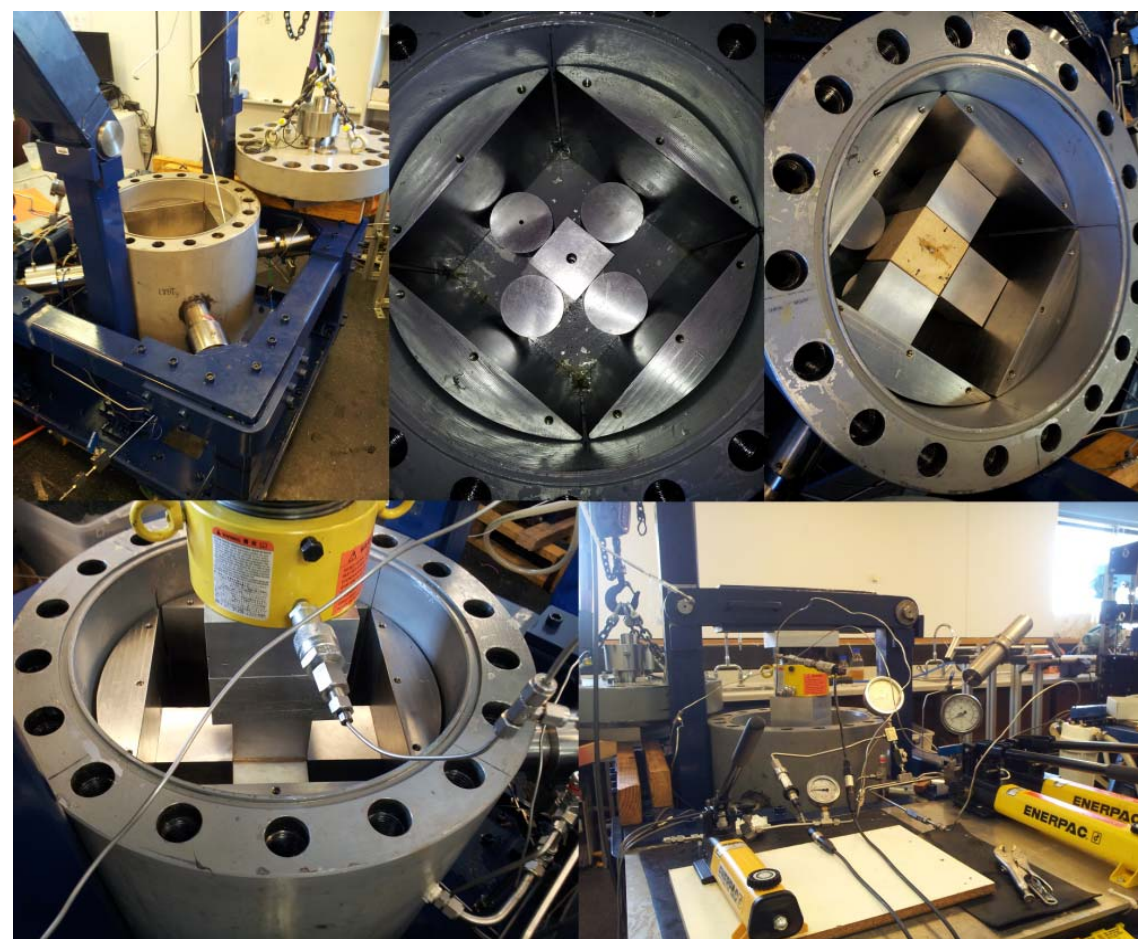

Figure 2: The TTSC shown from unloaded through to sample loading and pressure lines connected and ready for hydraulic fracturing testing to start.

In Figure 2 a view of the TTSC and the process of loading a $100 \mathrm{~mm}$ cube sample are shown. The stresses applied in the vertical and in both horizontal directions can be controlled individually and changed real-time while performing a fracturing test. The longer of the metal rods sealed in to the sample is 
connected to the hydraulic fracturing fluid chamber below the TTSC. This chamber is loaded with a fracturing fluid with a viscosity of 100,000 $\mathrm{cP}$ which is then pressurised to flow in to the open hole area of the sample and begin the fracturing process. Such large fluid viscosity is necessary to be used for a small size sample at the lab scale to propagate the fracture very slowly, hence provide adequate time for recording pressure-time data. The required viscosity to simulate field operation hydraulic fracturing is obtained through the scaling analysis [8].

\section{Test procedure}

With the sample loaded into the TTSC, pressures are then set in each direction individually. Applying large stress anisotropy is desired to help the fracture to initiate more easily. Stresses in each direction are simultaneously built up until the minimum horizontal stress is reached. The vertical and maximum horizontal stresses are then increased together until the desired stress levels are reached. The area of the piston that pressure was applied to by the pumps was smaller than the area of the sample which meant that the pressure on the sample was not equal to the pressure on the piston. The actual pressure applied to the pistons therefore needed to be calculated based on the desired pressure on the sample.

While the stress is being applied in one direction, it also increases the stress in the other directions due to the Poisson's effect, so it was important to relieve pressure on the minimum horizontal stress plane as the others were being increased. Manual PDP hand pumps were used to apply pressure for the principal stresses while an automatic high pressure syringe pump was used to control the pressure of the fracturing fluid. Pressure transducers were used to enable recording and monitoring of the pressures on the three stress directions as well as for the hydraulic fracturing injection fluid.

With the principal stresses set, the fracturing fluid was then injected at a high flow rate up to a constant pressure of 1200 psi. At this pressure, the flow rate was set to a low $7 \mathrm{cc} /$ hour until the sample fractured. It was important to maintain a low injection rate with a high viscosity fluid to allow for the fracture to open and propagate slowly towards the sample boundary. Slower propagation was desired in this experiment in particular to allow time for the fracture to reorient towards the direction of maximum stress once it had been initiated in the direction of minimum stress. When the test had concluded and a fracture had successfully propagated through the sample, all pressures were reduced back to atmospheric pressure, relieving stresses on the sample so it could be unloaded from the TTSC.

\section{Results and discussions}

Table 1 shows the magnitude of the three independent stresses applied to each sample and the pressure at which the hydraulic fracture initiated at the borehole wall. In the following sub-sections the produced results in each tested sample are presented and interpreted. 
Table 1: $\quad$ Principal stresses and fracture initiation pressure for each sample.

\begin{tabular}{|c|c|c|c|c|}
\hline Test Number & $\sigma_{\mathrm{V}}(\mathrm{psi})$ & $\sigma_{\mathrm{H}}(\mathrm{psi})$ & $\sigma_{\mathrm{h}}(\mathrm{psi})$ & Fracture Initiation P (psi) \\
\hline 1 & 2600 & 2000 & 1000 & 1900 \\
\hline 2 & 1600 & 1000 & 500 & 1720 \\
\hline 3 & 2400 & 2000 & 500 & 2310 \\
\hline 4 & 5000 & 3650 & 500 & 2450 \\
\hline
\end{tabular}

\subsection{Sample one}

In this sample it was expected that both fracture wings would open in the direction of maximum stress. As shown in Figure 3, one fracture wing did open in the expected direction while the other wing unexpectedly opened on an angle approximately $45^{\circ}$ to the direction of maximum stress. There could have been a variety of reasons for this occurring but it was most likely due to an inhomogeneity along the wellbore in that particular area of the sample which made fracturing in that direction more favourable. It is known from previous studies that the fracture will be influenced by inhomogeneities in low stress anisotropy which is what appears to have been the case in this test.

It can be seen from the results that towards the boundary of the sample, the fracture began to turn towards the direction of maximum stress as was expected for a fracture initially in different direction. Although this was not the planned or expected result, it provided good evidence that a fracture will begin to curve towards the direction of maximum stress even if it is initiated in a different direction at the wellbore. In this case, a larger sample size would have been ideal to allow the fracture more time to curve back to the direction of maximum stress.

The pressure-time curve plotted in Figure 4 shows the build up of pressure in the open hole area of the sample. The flow rate had been decreased when the expected fracture initiation pressure was being approached which is why the build up gradient decreased at approximately 1200 psi and again at 1450 psi.

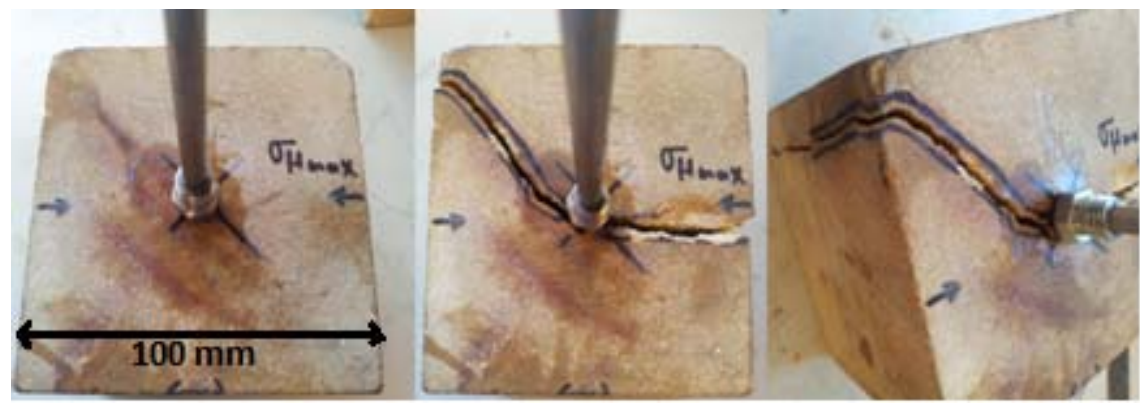

Figure 3: View of sample one after HF test: one fracture wing was not along the maximum stress direction. 


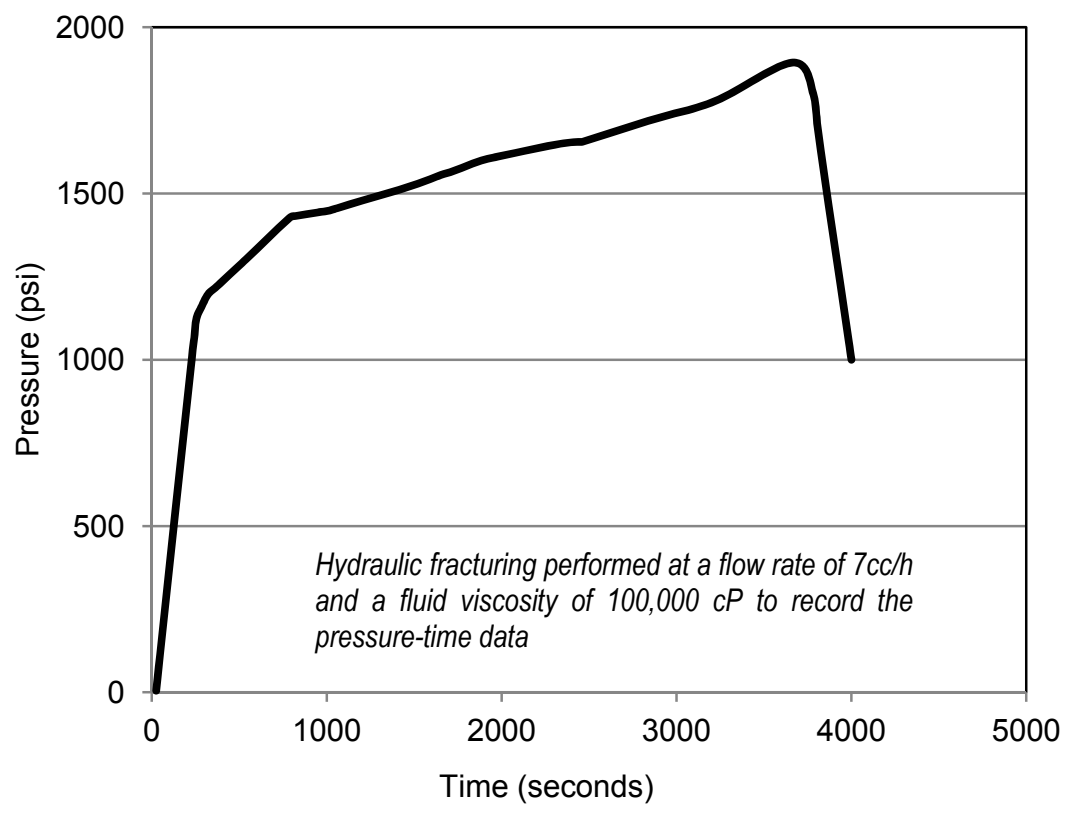

Figure 4: Pressure-Time curve showing the pressure build up of the fracturing fluid before the fracture initiation pressure.

Once the pressure reached $1900 \mathrm{psi}$, the pressure quickly declined which is a sign that the fracture had propagated to the boundary of the sample and testing had been completed.

\subsection{Sample two}

For sample two, the expectation was that fractures would initiate where the notches were created at the direction of minimum stress, and then curve back to the direction of maximum stress. As can be seen from the sample results displayed in Figure 4, the fracture failed to open in the direction of minimum stress and was instead opened in the direction of maximum stress. This is likely due to the notches created not being deep enough so the fracturing fluid pressure required to overcome the tangential stress at the notches was greater than the stress required to fracture the rock along the direction of maximum stress, hence the fracture propagated in this direction. The resulting fracture was very clean and did not deviate from the direction of maximum stress. This sample showing a regular fracture in the direction of maximum stress can be used as a base case for comparison with other samples of different fracture direction.

\subsection{Sample three}

This was in fact sample two which was used for a second hydraulic fracturing test as per preparation explained in the previous section. In this test the open 
fracture plane at $90^{\circ}$ to the direction of maximum stress was partially used as a notch for this sample. The resulting new fracture, as pictured in Figure 5, shows that the new fracture opened in the direction of minimum stress as desired, but did not curve back towards the direction of maximum stress. The fracture crossed over and in some sections joined up with the glued previous fracture and re-opened it. This was due to the glued section acting as an inhomogeneity which therefore affected the propagation direction of the fracture throughout the sample.

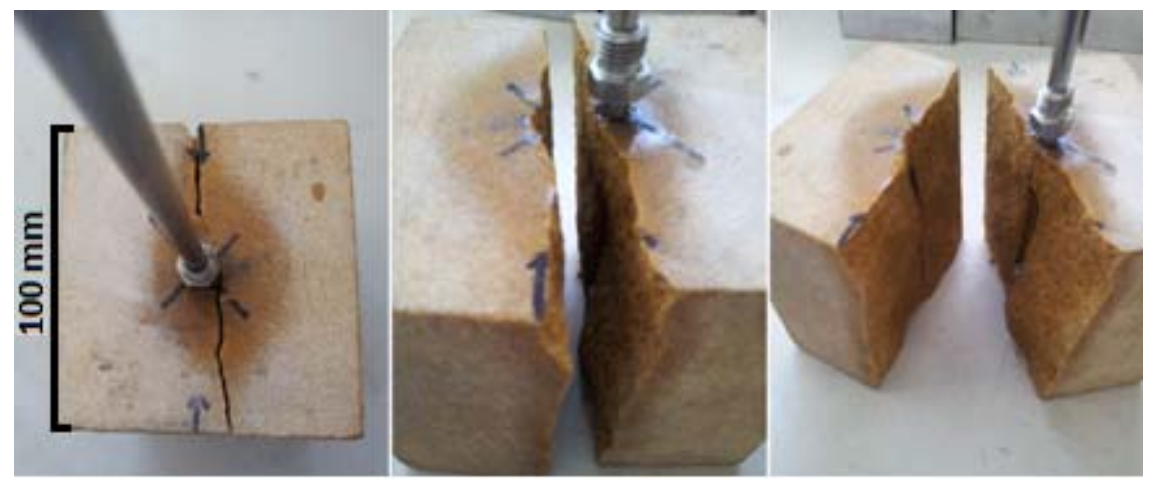

Figure 5: View of sample two after HF test: the fracture opened along the maximum stress direction.

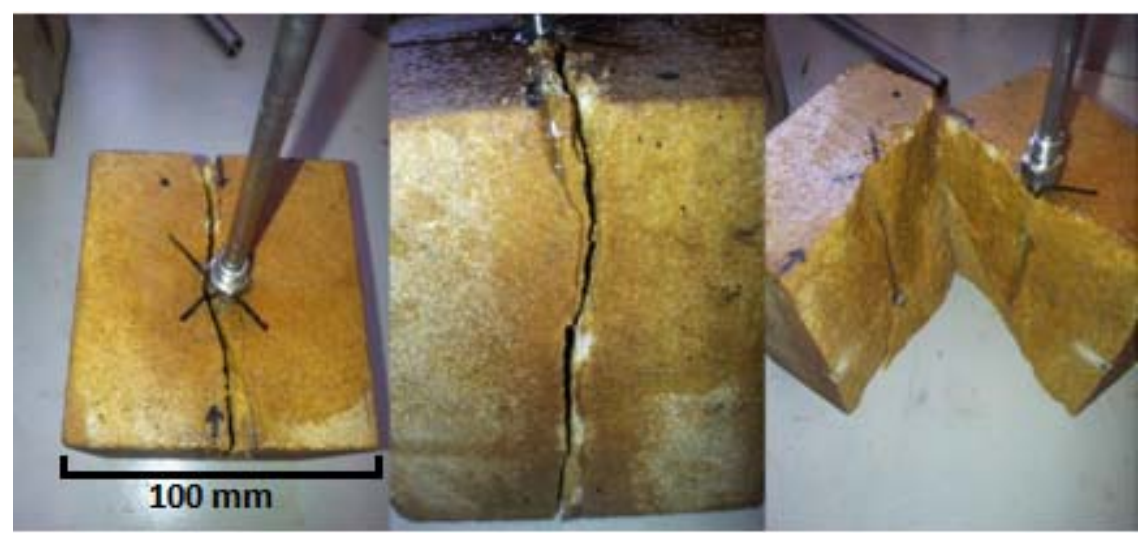

Figure 6: View of sample three after HF test: a new fracture created alongside the previously opened fracture from sample two.

The results observed demonstrated the importance of an existing natural fracture in the results of a hydraulic fracturing test. It was again noted that for future testing a larger sample size would be idea to allow the fracture more time and space to be able to curve back to the maximum stress direction. The rock sample is brittle and so once the fracture initiates, it quickly travels to a boundary 
which limits the time for the fracture to react to the principal stresses and curve to the direction of maximum stress.

\subsection{Sample four}

In this sample, where a deep notch was made at $45^{\circ}$ to the direction of maximum stress, a large stress anisotropy was set in order to reduce the effect of any inhomogeneity in the sample on the fracture propagation. It was expected that the fractures would open at an angle of $45^{\circ}$ to the direction of maximum stress where notches were created and then curve back to align with the direction of maximum stress. As can be seen from the images of the fractured sample in Figure 4, the fracture wings initiate from the location a notch but in both cases they quickly curve back to the direction of maximum stress towards the boundary of the sample.

The results from this test help prove that if a fracture is being affected by an inhomogeneity in the formation, once propagated away from the wellbore wall and senses the far field stresses it will eventually reorient itself towards the direction of maximum stress regardless of the local effects around the borehole due to sample inhomogeneity or existence of natural fractures. It can also be seen that the fractures tend to deviate in the rock in areas away from the wellbore which is due to inhomogeneities. After passing through these inhomogeneities, the fracture will respond to the principal stresses and rotate back to the direction of maximum stress.

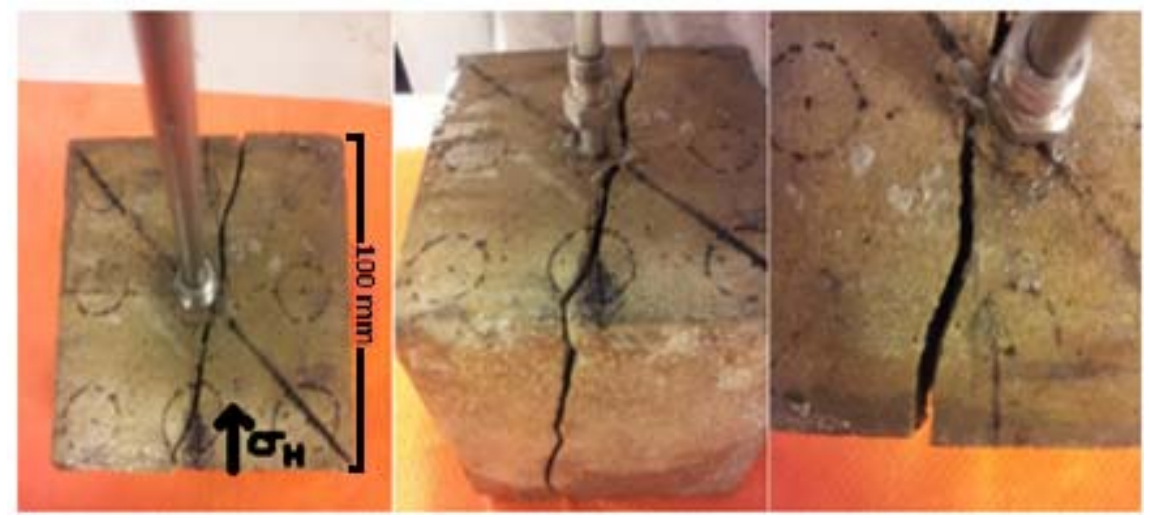

Figure 7: View of sample four after HF test: two deviated fracture wings that curve back to the direction of maximum stress.

\section{Conclusions}

This paper examined the outcomes of hydraulic fracture tests on three $100 \mathrm{~mm}$ tightly consolidated sandstone cube samples and one $100 \mathrm{~mm}$ cement cube sample. The results of these experiments have shown that a hydraulic fracture will tend to propagate and reorient in a direction which is perpendicular to 
minimum stress after overcoming effects from inhomogeneities both at the wellbore wall and throughout the formation. The complexity of hydraulic fracturing operations was apparent in these tests as even in a highly controlled laboratory environment, some fractures unexpectedly opened and propagated in directions different to that of the maximum stress direction.

The results have also indicated that on a small scale, a fracture will deviate due to the effects of inhomogeneities or natural fractures that are either preexisting in the formation or have been created from drilling and completions operations. On a larger scale and for field applications, it can be seen the fracture would curve and reorient towards the direction of maximum stress after the effects of inhomogeneities have caused a change in propagation direction.

\section{References}

[1] Valko, P. and Economides, M.J., Hydraulic Fracture Mechanics, Chichester: John Wiley \& Sons, 1995.

[2] Hossain, M.M., Rahman, M.K. and Rahman, S.S., Hydraulic fracture initiation and propagation: roles of wellbore trajectory, perforation and stress regimes. Journal of Petroleum Science and Engineering 27, pp. 129149, 2000.

[3] Nabipour, A., Evans, B. and Sarmadivaleh, M., Active Monitoring of a Hydraulic Fracture Propagation: Experimental and Numerical Study. APPEA Journal 2011, pp. 479-486, 2011.

[4] Yew, C., Mechanics of Hydraulic Fracturing, Houston: Gulf Publishing Company, 1997.

[5] Rasouli, V., Sarmadivaleh, M. and Nabipour, A., Some Challenges in Hydraulic Fracturing of Tight Gas Reservoirs: An Experimental Study. APPEA Journal 2011, pp. 499-506, 2011.

[6] Hudson, J. and Harrison, J., Engineering Rock Mechanics - An Introduction to the Principles, Oxford: Elsevier Ltd, 1997.

[7] Evans, B and Rasouli, V., A True Triaxial Stress Cell to Simulate Deep Downhole Drilling Conditions. APPEA Journal 2010, pp. 61-70, 2010.

[8] Sarmadivaleh, M. Experimental and Numerical Study of Interaction of a Pre-Existing Natural Interface and an Induced Hydraulic Fracture, $P h D$ thesis, Curtin University, 2012. 